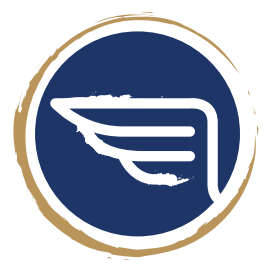

\title{
El precio de la verdad
}

\section{Germán Scalzo}

Universidad Panamericana

gscalzo@up.edu.mx

Primer premio ex aequo XI Foro Universitas 2019:

"Fe, razón y cultura", concurso intercampi de ensayos, Universidad Panamericana. 


\title{
"Scientia donum Dei est, unde vendi non potest"
}

\author{
Proverbio Medieval
}

\section{Introducción}

Poco antes de su muerte, Max Weber confiesa en un par de conferencias ${ }^{1}$ la inmensa especialización que observaba en el ámbito científico-académico, así como su progresiva instrumentalización: "como un producto que se elabora en una fábrica" ${ }^{2}$, y en especial una actitud mercantil extraña al espíritu universitario, que grafica con el incipiente pero ya patente pragmatismo norteamericano: “[e]l joven norteamericano no siente respeto por nadie ni por nada (...) frente al profesor que tiene delante, el muchacho norteamericano piensa que le está vendiendo sus conocimientos y sus métodos a cambio del dinero de su padre, exactamente de la misma manera que la verdulera le vende a su madre una col. Eso es todo"'s.

Un siglo más tarde, la mentalidad pragmatista que denunciaba el sociólogo alemán ha convertido a la institución universitaria en una business university que propone (o más bien, impone) un cierto ideal de "excelencia" como reemplazo a la cultura ${ }^{4}$. Para Readings, el concepto de "excelencia" esconde la crisis de la universidad contemporáneas: "el llamado a la excelencia no muestra otra cosa que el hecho de que ya no hay ninguna idea de Universidad, o más bien, que esa idea ha perdido todo su contenido". Alejandro Llano coincide con este diagnóstico alarmante: "[1]a Universidad que, desde hace ocho siglos, ha sido capaz de responder a los desafíos provenientes del exterior, se muestra ahora inerme ante la amenaza que brota de ella misma y que la está vaciando de su propio contenido (...) se trata de una decisiva encrucijada institucional. Lo que le sobra a la Universidad es organización; lo que le falta es vida"'.

Este ensayo pretende contribuir al debate sobre cómo revitalizar a la Universidad tras el “desencantamiento religioso" que-según Weber-produjo el "proceso de racionalización"8 occidental,

1. Se trata de La ciencia como vocación (1917) y La política como vocación (1919). Ver Max Weber, El político y el científico (Madrid: Alianza, 1967).

2. Weber, El político y el científico, 207.

3. Weber, El político y el científico, 218-219.

4. "Podemos escribir excelencia en el centro del diagrama en donde alguna vez estuvo cultura". Bill Readings, The University in Ruins (Cambridge: Harvard University Press, 1996), 117.

5. Readings, The University in Ruins, 86.

6. Readings, The University in Ruins, 39.

7. Alejandro Llano, Universidad y unidad de vida según san Josemaría Escrivá (Pamplona: Universidad de Navarra, 2002), 5. El destacado es propio.

8. Sobre el proceso de racionalización como unidad temática de la obra weberiana véase: Friedrich Tenbruck, "The problem of thematic unity in the works of Max Weber", British Journal of Sociology 31, n. o 3 (1980): 316-351. "La conclusión del proceso de desencantamiento religioso provee el espíritu desde el cual el capitalismo desempeña su rol como la fuerza racionalizadora de la modernidad. La emergencia de este último estadio de racionalidad es llevado a cabo por nuevos agentes: la ciencia, la economía y la política", Tenbruck, “The problem of thematic...”, 322. 
y cuyo hito principal es la caída del "gran mito" (el catolicismo) como fundamento unificador de la realidad, para ser sustituido por la racionalidad científica. De modo más concreto, se quiere mostrar la importancia de la Universidad en la humanización y unificación de las culturas, y cómo ésta se vuelve instrumental a los intereses de turno cuando pierde el ideal de unidad que la caracterizó desde sus mismos inicios.

La reflexión sobre el sentido de la cultura y la finalidad de la vida humana permitirá mostrar que la sociedad no puede prescindir de un ámbito de "humanización" como el que ofrece la Universidad. Sin embargo, si esta unidad no está fundamentada en la verdad, la racionalidad no solo acaba creando una "jaula de hierro" que limita la libertad, sino que además cae en la irresoluble tensión epistemológica del politeísmo de valores, que, paradójicamente, en aras del progreso, supone un retroceso al mundo mítico, como agudamente destacó el sociólogo alemán: "los numerosos dioses antiguos, desencantados y, por tanto, convertidos en poderes impersonales, salen de sus tumbas, quieren dominar nuestras vidas y reanudan entre ellos su eterna lucha"10.

\section{El hombre y la cultura}

Recientemente, tras varios siglos de predominio racionalista inspirado por el espíritu de la Ilustración, se ha prestado mayor atención a la dimensión cultural de la vida de los hombres, lo cual ha propiciado un retorno a los fundamentos antropológicos para explicar el sentido de la vida humana ${ }^{11}$. El proyecto moderno de racionalidad, inspirado por la mentalidad de progreso de la Ilustración, se había propuesto dar nuevas razones para explicar lo humano, al margen de la fe, dejando sin respuesta a las preguntas fundamentales sobre el sentido de la vida. Estas preguntas aún siguen abiertas, y para responderlas -claro está, no de modo definitivo, sino vital- es preciso volver la vista hacia atrás, y apreciar lo que la humanidad ha reconocido como valioso y perenne a lo largo de los siglos, esto es, la visión de los clásicos.

La vida humana se desarrolla en interacción con un entorno biológico y cultural que le viene dado, pero cuya adaptación al mismo no es necesaria - como es el caso de los demás seres creados- sino libre; en otras palabras, el hombre tiene la capacidad -y el deber- de decidir la manera de relacionarse con su entorno. Esta especie de apertura o indeterminación que supera lo biológico es señal de que

9. Max Weber, La ética protestante y el espíritu del capitalismo, ed. por Francisco Gil Villegas (México: Fondo de Cultura Económica, 2003).

10. Weber, El político y el cientifico, 217-8.

11. Alasdair MacIntyre, Dependent Rational Animals. Why Human Beings Need the Virtues (Chicago: Open Court, 1999). Marcel Mauss, Ensayo sobre el don. Forma y función del intercambio en las sociedades arcaicas (Buenos Aires: Katz Editores, 2009). 
sin la dimensión cultural la vida del hombre no sería viable, en otras palabras, que necesita vivir en una cultura' ${ }^{12}$ "El hombre no está en el mundo como lo está el animal; más que adaptarse al medio en el que vive, adapta el medio a sus necesidades, lo transforma, lo moldea. Para ello cuenta con dos instrumentos de los que no dispone el animal: manus et lingua"13. Así, las manifestaciones básicas de la cultura -y en cierto sentido lo más propio del hombre- son la palabra y la técnica, y es en relación a ellas como cada hombre descubre, de modo concreto e histórico, el sentido y la finalidad de su vida.

La cultura ${ }^{14}$, o, mejor dicho, las culturas son producto de la interacción histórica de los distintos grupos humanos con la naturaleza, en la que el hombre no solo se procura los medios materiales para vivir bien, sino que descubre el orden inscrito en su propio espíritu, es decir, su capacidad de avanzar en el conocimiento de la verdad inmutable. Es gracias al trabajo humano, continuada interrelación cultural e histórica entre todos los hombres de todos los tiempos, que se desvela la riqueza oculta en la naturaleza, sus potencialidades ocultas. Pero más importante, es gracias al trabajo que el hombre se descubre a sí mismo, pone en acto lo más valioso de su acto de ser: su libertad, su capacidad de conocer y amar, lo cual le otorga una dignidad especial en la Creación ${ }^{15}$.

El origen de la cultura no reside en la naturaleza sino en la libertad, en el acto de conocer y querer del espíritu humano ${ }^{16}$ : es precisamente gracias a los incontables modos de ser y hacer del hombre, que la cultura está abierta a la riqueza de la diversidad, pues los rasgos espirituales y materiales, internos y externos, que caracterizan los modos de vida de los distintos grupos humanos, son innumerables ${ }^{17}$. La variedad de culturas y los diversos modos de vida dentro de cada una de ellas pone de manifiesto que el fin del hombre no es natural - no viene dado- sino libre, es decir, que tiene que descubrirlo, en relación con los demás. Más aún, la dependencia intergeneracional sugiere que su fin está íntimamente unido

12. Ver Arnold Gehlen, Antropología filosófica. Del encuentro y descubrimiento del hombre por sí mismo (Barcelona: Paidós, 1993).

13. Carlos Goñi, Ética borrosa. Sobre la necesidad de la reflexión y el silencio (Madrid: Palabra, 2010), 167.

14. "Con la palabra cultura se indica, en sentido general, todo aquello con lo que el hombre afina y desarrolla sus innumerables cualidades espirituales y corporales, procura someter el mismo orbe terrestre con su conocimiento y trabajo; hace más humana la vida social, tanto en la familia como en toda la sociedad civil, mediante el progreso de las costumbres e instituciones; finalmente, a través del tiempo expresa, comunica y conserva en sus obras grandes experiencias espirituales y aspiraciones para que sirvan de provecho a muchos, e incluso a todo el género humano", Pablo VI, Vaticano II, Constitución pastoral Gaudium et Spes (7 de diciembre de 1965), n. 53.

15. CEM, Catecismo de la Iglesia Católica (México: CEM, 2012), n. 355 a 358. El hombre es la "única criatura en la tierra a la que Dios amó por sí misma” (GS 24,3), “capaz de conocer y amar a su Creador” (GS 12,3). Ver Miguel Alfonso Martínez Echevarría y Germán Scalzo, “Trabajo, don, cultura y economía: Hacia un nuevo enfoque del problema económico", Cuadernos Salmantinos de Filosofía 47, (2020): 379-399.

16. Benedicto XVI, La búsqueda de Dios, fundamento de toda cultura (París: Discurso al mundo de la cultura, 2008). En este "discurso al mundo de la cultura", el papa emérito no solo resalta que la búsqueda de Dios es el fundamento de toda cultura, sino también la importancia que tiene la palabra en este proceso: "La búsqueda de Dios requiere, pues, por intrínseca exigencia una cultura de la palabra", p. 5.

17. Ver Cliford Geertz, The interpretation of Cultures (New York: Basic Books, 1973). 
al fin de toda la humanidad, lo cual reclama algún fundamento de unidad y manifiesta una relación con el fenómeno religioso ${ }^{18}$, el modo en que el hombre percibe y entiende el sentido de lo sagrado, y que recibe en forma de mito ${ }^{19}$. La unidad como ideal tiene implicancias éticas y escatológicas ${ }^{20}$, pues "cada hombre individual, en cuanto persona, tiene la posibilidad de la perfección, o sea, de la infinitud positiva, precisamente en la capacidad de entenderlo todo con su razón y de abrazar todo con su corazón, o de entrar en una unidad viva con todo"21.

\section{La unidad como ideal}

Una cultura se fundamenta en que existe en las personas una tendencia a progresar, a vivir mejor. Esta tendencia trasciende el tiempo y el espacio; no solo se apoya en los avances de las generaciones anteriores, sino que a lo largo de la historia las culturas superiores se imponen a las inferiores ${ }^{22}$, dando lugar a un proceso de creciente unificación. Aunque este rasgo de unificación cultural siempre ha existido, se ha hecho más patente en los últimos años, por la globalización y la aceleración de la tecnología. De modo especial en el último siglo, los nuevos medios de comunicación y producción han ido creado un mundo en el que las distintas culturas, al menos en su aspecto externo, de alguna manera se han ido homogeneizando, apareciendo modos de comportarse que parecen ser universales.

En el ámbito universitario este fenómeno ha sido notable en la unificación de los programas y planes de estudio a nivel mundial. A modo de ejemplo, la tendencia actual es que un alumno pueda cursar un semestre académico en una universidad remota, recibiendo los mismos conocimientos que en su universidad de origen, lo cual significa evitar todas aquellas cuestiones culturales -en realidad su vertiente normativa- que no pueden ser universalmente aceptadas. Sin dudas, esta experiencia es enriquecedora y puede contribuir a una mejor integración cultural, pero lo que se quiere destacar es que tal unificación no puede ser más que aparente o superficial. Las manifestaciones de la cultura-las técnicas o el lenguaje- no pueden ser un principio de unificación; se requiere algo más profundo, que remita al origen ${ }^{23}$.

18. Ver Philip Rieff, Charisma. The Gift of Grace, and How It has Been Taken Away from Us (New York: Pantheon Books, 2007) y Franz Steiner, Taboo (London: Cohen and West, 1956).

19. Ver Josef Pieper, Sobre los mitos platónicos (Barcelona: Herder, 1984).

20. Benedicto XVI, La búsqueda de Dios..., 5.

21. Vladímir Soloviov, La justificación del bien. Ensayo de filosofía moral (Salamanca: Ediciones Sígueme, 2002 ), 253. 22. Al momento de comparar culturas, tarea nada fácil, conviene evitar hacerlo desde el éxito material o los resultados obtenidos, que si bien pueden ser señal de bienestar, no necesariamente contribuyen a la unidad o al verdadero desarrollo de dicha cultura. "Entre los criterios que determinan el valor de una cultura, están, en primer lugar, el significado de la persona humana, su libertad, su dignidad, su sentido de responsabilidad, y su apertura a la trascendencia", Juan Pablo II, Constitución Apostólica Ex corde Ecclesiae (1990), n. ${ }^{\circ} 45$.

23.Martínez Echevarría y Scalzo, "Trabajo, don, cultura ...". 
La advertencia ante la falsa tendencia a la unidad entre todos los hombres por medio de la técnica ya estaba presente en la construcción de la "torre de Babel", simbolizada por los ladrillos y la brea ${ }^{24}$. El relato bíblico recoge una tentación que ha estado siempre en el corazón humano: "[e]difiquemos una ciudad y una torre cuya cúspide llegue hasta el cielo. Hagámonos así famosos y no andemos más dispersos sobre la faz de la Tierra" ${ }^{25}$. Sin embargo, el resultado de esta empresa fue el contrario al que se pretendía: la confusión de las lenguas ${ }^{26}$, la fragmentación e incomunicación entre los hombres. En el seno de la tradición judía en que aparece este relato se pone de manifiesto que la unidad de las culturas no puede plantearse como rebelión contra Dios, pero implícitamente que es la existencia de una verdad absoluta el único fundamento posible de la comunidad universal. El recurso exclusivo a la racionalidad técnica como factor de unidad conduce a una mayor dispersión, a pesar de su aparente uniformidad exterior.

Conviene distinguir entre la unidad del mundo exterior - una especie de artefacto externo que el hombre va construyendo con su progresivo conocimiento de la ciencia y la técnica- y la unidad que se va alcanzando en la comunión con la verdad. La racionalidad ilustrada ha ido extendiendo la idea de que el desarrollo de la ciencia y la técnica siguen un camino único y lineal, y que es a través de su avance continuado como la ciencia por sí misma puede conocer -y dominar- la realidad, sin necesidad de las humanidades. Lo cierto es que, a medida que avanza la técnica, la relación entre los efectos buenos y malos se hace más cada vez compleja, y cada vez más difícil de resolver técnicamente. El ámbito universitario es un ejemplo de ello: cuanto más complejos y utilitarios se vuelven los saberes, no solo aumenta la ansiedad ante la imposibilidad de dominarlos, sino que cada vez más crece la percepción de su inutilidad, es decir, de que realmente sirvan para algo ${ }^{27}$.

De alguna u otra manera todas las culturas expresan la tendencia innata de todo hombre hacia la verdad, el bien y la belleza, que, junto con la unidad, constituyen los llamados trascendentales del ser. El cristianismo es la respuesta divina a esa tendencia natural del hombre, una respuesta que se realiza en la medida que toda cultura se abre al misterio de Cristo, "camino, verdad y vida" 28 , y el único fundamento posible de la unidad del género humano. El reconocimiento de la verdad es el primer paso en la construcción de una verdadera comunidad universal; sin el respeto a la verdad-que está más allá de la técnica- se hace imposible la creciente unidad-diversidad de las culturas. Así, la unidad

24. Martínez Echevarría y Scalzo, "Trabajo, don, cultura ...”, 390.

25. Gén 11,4.

26. En hebreo " $b l-b l$ " significa balbuceo, confusión al expresarse algo.

27. Miguel Alfonso Martínez Echevarría, "El modo universitario de entender la economía”, Nuevas Tendencias, $\mathrm{n}^{\circ} 93$ (2014): 3-15.

28. Jn 14,6 . 
del género humano sólo puede lograrse mediante el desarrollo de una cultura superior surgida del reconocimiento de la verdad. Esa cultura superior no lleva a una unidad homogénea, sino que respeta y potencia la originalidad propia de cada pueblo y la libertad de cada hombre, con el único requisito de que respete la dignidad humana y no se cierre a la posibilidad de una verdad eterna y trascendente.

Cuando la cultura crece en torno a la verdad prepara para la misma realización plena de esa libertad, la unión de todo hombre con Dios, que sólo se realizará si acepta libremente a Cristo, don de Dios a los hombres ${ }^{29}$. El cristianismo no es un fenómeno religioso que da origen a una cultura singular, ni es fruto de la reflexión racional sobre lo divino, sino que es la misma presencia de Dios sobre la tierra, hecho hombre. Con la Encarnación Dios ha unido a todos los hombres en la humanidad de Cristo, misterio que se manifestará plenamente al final de los tiempos. Cristo, perfecto Dios y perfecto hombre, impulsa a toda la humanidad a su perfección, y lo hace respetando la libertad y creatividad de todos los hombres, poniendo de manifiesto que cuenta con la historia y con la diversidad de las culturas $^{30}$.

La institución universitaria ocupa un rol fundamental e insustituible en ese proceso, pues -como señala Llano- "la esencia de la Universidad estriba en la convicción de que esa unidad orgánica es posible, de que existe una articulación necesaria entre verdad y unidad que puede ser desvelada por la más alta capacidad humana, por la teoría o contemplación serena de la realidad"31.

\section{La misión de la universidad}

Fueron precisamente los cristianos quienes, libremente y animados por la tendencia natural a alcanzar la perfección que les es posible, tanto a nivel personal como social, fundaron las universidades en la edad media para cultivar lo más alto de la reflexión humana ${ }^{32}$ : la búsqueda de la verdad. La verdad no es - no puede ser- impuesta sino que comparece cuando los mejores hombres de cada cultura

29. Para el cristianismo, la verdad es una síntesis de la noción griega de verdad ( $\dot{\alpha} \lambda \hat{\eta} \theta \varepsilon 1 \alpha$, aletheia) y de la hebrea (אמא, emet), por lo que es tanto evidencia racional como revelación entrañable de una autoridad. "La reconciliación del hombre en un solo cuerpo con Dios y la inclusión de la humanidad entera en la promesa universalizan el emet hebreo: la verdad, que proviene de Dios, y que ha sido no sólo revelada por Él, sino también testimoniada con su vida, muerte y resurrección, debe ser conocida y vivida por todos los hombres", José María Llovet Abascal, "Aletheia, emet y veritas", Revista humanidades. Foro Universitas, (2013): 33.

30. Joseph Ratzinger, Introducción al Cristianismo (Salamanca: Ediciones Sígueme, 2001).

31. Llano, Universidad y unidad..., 12. Continúa Llano: "En cambio, la contraposición entre espíritu y materia, entre verdad y eficacia, entre educación humanística y capacitación profesional, es la herida no restañada por la que se desintegra el espíritu universitario".

32. Ver Higinio Marín, "La universidad como excepción”, en El hombre y sus alrededores, (Ediciones Cristiandad, 2013) y Juan Pablo II, Constitución Apostólica..., punto 1: "Por su vocación la Universitas magistrorum et scholarium se consagra a la investigación, a la enseñanza y a la formación de los estudiantes, libremente reunidos con sus maestros animados todos por el mismo amor del saber”. 
profundizan en el conocimiento de la realidad, y lo comparten con los demás. No es posible acceder a la verdad, y por tanto, alcanzar la plenitud humana - de toda la humanidad-, y cultural -como anticipación del Reino de Dios- con el mero alcance de la razón, sino que se necesita además de la fe, así como de la libre aceptación del don de Dios. Como nos recuerda Juan Pablo II, "la fe y la razón (fides et ratio) son como las dos alas con las cuales el espíritu humano se eleva hacia la contemplación de la verdad. Dios ha puesto en el corazón del hombre el deseo de conocer la verdad y, en definitiva, de conocerle a Él para que, conociéndolo y amándolo, pueda alcanzar también la plena verdad sobre sí mismo" $"$ 33.

Aunque la búsqueda de la verdad ya estaba presente en los mismos inicios de la reflexión filosófica ${ }^{34}$, alcanza su plenitud con la Revelación, pues la razón por sí misma no puede llegar al conocimiento pleno de la Verdad. La Universidad es el estandarte cultural de esa empresa común que une a toda la humanidad en su empeño por participar de su propia liberación ${ }^{35}$. Como destaca Llano, “[n]o es un acontecimiento histórico contingente el hecho de que la Universidad sea una institución original y originariamente cristiana" 36 (específicamente, católica), pues el catolicismo permite "unificar existencialmente en el trabajo intelectual dos órdenes de realidades que muy a menudo se tiende a oponer como si fuesen antitéticas: la búsqueda de la verdad y la certeza de conocer ya la fuente de la verdad"37.

Sin embargo, la armonía entre razón y fe que habían realizado los Padres de la Iglesia con la síntesis entre emet y aletheia, se rompe con el protestantismo, que continúa la tradición hebrea de la verdad en cuanto emet. Sin embargo, “[e]l emet no es toda la verdad: la razón puede descubrir muchas verdades que no están contenidas en el emet, y si la fe no es capaz de integrarlas y darles sentido, entonces estas verdades racionales terminan por edificar una visión independiente del mundo que tiene su propia praxis y su propia finalidad. Por ello, cuando Lutero separó nuevamente emet y à $\lambda \hat{\theta} \theta \varepsilon 1 \alpha, \mathrm{y}$ declaró que con la helenización del cristianismo había comenzado su perversión, inauguró con ello la modernidad." ${ }^{38}$ Como consecuencia, esta actitud cae primero en una especie de fideísmo, y con el correr de los años en una mezcla de progresismo y evolucionismo, una actitud de "no creer en nada y poder hacerlo todo", que es lo que se observa en el diagnóstico de Weber con el que se introduce este ensayo.

33. Juan Pablo II, Fe y razón (México, 2000), introducción.

34. La cuestión que se suscita entre Sócrates y los sofistas es ejemplo de que esta tensión estaba presente en los mismos orígenes de la filosofía. Ver, por ejemplo, Marcel Hénaff, Le prix de la verité: le don, l'argent, la philosophie (París: Du Seuil, 2002), y Marcel Detienne, The Masters of Truth in Archaic Greece (New York: Zone Books, 1996).

35. "Conocerán la verdad, y la verdad los hará libres", Jn 8,32.

36. Llano, Universidad y unidad..., 8.

37. Juan Pablo II. Discurso a los profesores y alumnos de la Universidad Luiss de Roma (17 noviembre de 1998), punto 2.

38. Llovet Abascal, "Aletheia, emet y veritas", 37. 
Es precisamente por esto que la Universidad -en su sentido más auténtico-adquiere un rol fundamental en el camino hacia la unidad a través de la profundización y el diálogo de los saberes en torno a la verdad $^{39}$. Como se ha dicho, el ideal universitario de la unidad, no solo ha estado presente en el mismo origen católico de la institución universitaria, sino que ha animado su espíritu a lo largo de todos estos $\operatorname{siglos}^{40}$. "La fuente de su unidad deriva de su común consagración a la verdad, de la idéntica visión de la dignidad humana y, en último análisis, de la persona y del mensaje de Cristo que da a la Institución su carácter distintivo"41. Es por su continua e incansable labor de cultivo del saber y búsqueda de la verdad $^{42}$ que la fe católica concede un nuevo y más hondo sentido a las obras del propio trabajo y a la humanización de las culturas. "La fe se hace cultura porque enseña a amar al hombre en su concreta humanidad, en esa unidad vital que está hecha de materia y espíritu, de intimidad y trascendencia, de singularidad irrepetible y de apertura a lo universal" ${ }^{43}$.

\section{Conclusión}

Aunque la respuesta a la cuestión sobre el "precio de la verdad" ha estado ante nuestros ojos desde el principio, en el epígrafe, ha sido necesario este breve desarrollo para comprenderla mejor. Ésta es precisamente la actitud universitaria: dar muestras de razón que permitan un acercamiento a la realidad de las cosas -que por ser verdaderas, buenas y bellas- dejan abierto el camino al Ser con mayúsculas.

No es necesario "innovar" para encontrar respuesta a ésta y otras preguntas fundamentales, sino acudir a la sabiduría que hemos heredado de nuestros antepasados y especialmente buscar "la novedad" en las enseñanzas básicas del cristianismo -como síntesis razonada de la fe en torno a la verdad revelada-. Esta sabiduría nos permite diferenciar entre valor y precio ${ }^{44}$, al recordarnos una y

39. Juan Luis Lorda, La vida intelectual en la Universidad. Fundamentos, experiencias y libros (Pamplona: EUNSA, 2016), 58: "El cristianismo es connatural con la idea de que el saber tiene unidad, porque toda la realidad procede de una causa inteligente, de un solo Dios (...) De manera que tanto el presupuesto como el fin de las tareas universitarias es la existencia de Dios".

40. Lorda, La vida intelectual..., 53-75. Ver también Juan Pablo II, Constitución Apostólica..., y Llano, Universidad y unidad... 41. Juan Pablo II, Constitución Apostólica..., punto 21. (había puesto 1990 pero presiento que era 1998).

42. "En efecto, la institución universitaria, que nació del corazón de la Iglesia, se ha caracterizado a lo largo de los siglos por el cultivo del saber y la búsqueda asidua de la verdad al servicio del bien del hombre. La investigación científica (...) [j]amás puede ignorar la dimensión humanística, que corresponde a la universidad en el ámbito de la profundización del saber, de su adecuada transmisión y de su insustituible misión educativa. En efecto, la universidad se sitúa en la tradición de la caritas intellectualis, en la que el saber y la experiencia del descubrimiento científico, al igual que la de la inspiración artística, se transforman en dones que se comunican como una gran energía. La fe cristiana reconoce en ello la verdadera sabiduría, don del Espíritu Santo” Juan Pablo II, Discurso a los profesores..., punto 2.

43. Llano, Universidad y unidad..., 8. 
otra vez que las cosas más importantes de la vida son un don recibido, gratuito, al que hay que saber corresponder. Precisamente por eso la verdad no tiene un precio económico aunque sí símbólico: la propia vida, la profunda transformación personal que supone el compromiso fiel a la verdad, y que se manifiesta en la entrega de sí a través de la santificación del trabajo. Ese es el "precio" que el joven rico no quiso pagar, y -como dice el Evangelio- "se fue triste" ${ }^{45}$.

Frente a una cultura predominante que nos impulsa hacia el resultado inmediato, esclavizándonos y fragmentándonos, el cristianismo nos dice que el hombre, constituido en un don gratuito, no se realiza -es decir, no se libera- en el éxito sino en la fidelidad a la verdad, que es una persona. La amorosa contemplación de la verdad puede ayudarnos a acoger el don con asombro y gratitud, con la actitud paciente y esperanzada de quien cultiva - origen de la palabra cultura- la naturaleza, como reacción al activismo - muchas veces estéril-al que nos empuja la business university. El crecimiento irrestricto que caracteriza al hombre ${ }^{46}$ es señal de que la verdad ciertamente puede ser un fin humano, pues nunca se alcanza de manera definitiva sino que siempre es posible descubrir novedad al profundizar en ella. Cuando la verdad, que se dirige a la interioridad de todos, es aceptada, puede ser trasmitida como algo vivo, permitiendo que el trabajo y la cultura sean comunes.

Es por eso que no existe un modelo de cultura sino más bien una misteriosa llamada universal a la unidad en Cristo, que es lo que hace que los cristianos, en el medio del mundo y desde culturas muy diversas, contribuyan a la construcción del Reino en la entrega generosa de sí, consecuencia de que han recibido la misión de servir, de dar a conocer a Cristo en el ejemplo del propio don. El encuentro personal con la verdad no solo es el fin de la vida universitaria sino también su raíz fecunda ${ }^{47}$, señal de que no es el resultado de la acción del hombre lo que verdaderamente lo hace libre, sino la apertur a interior de su espíritu a la trascendencia, como resume de manera magistral el lema que aúna nuestra labor panamericana: Ubi spiritus, libertas $^{48}$.

44. Obsérvese que el precio es en la actualidad la medida social por excelencia. Al preguntarse sobre el origen de la medida, Kula menciona que fue inventada por Caín, al observar que la ofrenda de su hermano agradaba más a Dios. Lo que se quiere destacar es que toda valoración humana remite en última instancia a lo sagrado, y, contrariamente a lo que piensa la ciencia económica moderna, la formación de los precios no es un mero proceso técnico, sino prudencial, estrechamente ligado a la recta valoración de las cosas. Ver Witold Kula, Las medidas y los hombres (Buenos Aires: Siglo XXI, 1980).

45. Mt 19:16. "La alegría es también una manifestación de la libertad de espíritu (...) sabernos libres para amar nos lleva a experimentar en el alma la alegría (...) Como dice el Papa Francisco, Él «es el autor de la alegría, el Creador de la alegría. Y esta alegría en el Espíritu nos da la verdadera libertad cristiana. Sin alegría, los cristianos no podemos ser libres: nos convertimos en esclavos de nuestras tristezas»", Fernando Ocariz, Carta pastoral (9 de enero de 2018 ), n. ${ }^{\circ} 6$. Debo esta cita al padre Coronel Salinas.

46. Leonado Polo, Quién es el hombre. Un espiritu en el tiempo (Madrid: Rialp, 1991).

47. "La Universidad sólo puede estar enfocada hacia la persona si hace de la causa de la Verdad su razón de ser, y pone al servicio de la misma todas sus fuerzas y medios.” José Ángel Agejas Esteban y Salvador Antuñano Alea, Universidad y persona: Una tradición renovada (Pamplona: EUNSA, 2019), 135.

48. "Al recopilar los ideales que pide nuestro lema, me queda el convencimiento, de estar persiguiendo un imposible, una utopía. Primer ideal: que en ese $u b i$, que es la universidad, haya libertad, es decir: personas que sean, que se sepan, 


\section{Bibliografía}

AAVV. La Biblia. España: San Pablo.

Agejas Esteban, José Ángel y Salvador Antuñano Alea. Universidad y persona:

Una tradición renovada. Pamplona: EUNSA, 2019.

Benedicto XVI. La búsqueda de Dios, fundamento de toda cultura. París: Discurso al mundo de la cultura, 2008.

CEM. Catecismo de la Iglesia Católica. México: CEM, 2012.

Coronel Salinas, José Antonio. “Nuestro lema: Ubi spiritus, libertas (Educación y libertad)”, 2018.

Detienne, Marcel. The Masters of Truth in Archaic Greece. New York: Zone Books, 1996.

Geertz, Cliford. The interpretation of Cultures. New York: Basic Books, 1973.

Gehlen, Arnold. Antropología filosófica. Del encuentro y descubrimiento del hombre por sí mismo. Barcelona: Paidós, 1993.

Goñi, Carlos. Ética borrosa. Sobre la necesidad de la reflexión y el silencio. Madrid: Palabra, 2010. Hénaff, Marcel. Le prix de la verité: le don, l'argent, la philosophie. París: Du Seuil, 2002.

Hoevel, Carlos. "Ante la llegada de la business university". Integra Educativa 8, n. 2 (2018): 59-80.

Juan Pablo II. Discurso a los profesores y alumnos de la Universidad Luiss de Roma, 17 noviembre de 1998.

Juan Pablo II. Constitución Apostólica Ex corde Ecclesiae. 1990.

Juan Pablo II. Fe y razón. México, 2000.

Kula, Witold. Las medidas y los hombres. Buenos Aires: Siglo XXI, 1980.

e incluso, que se sientan libres. Segundo: que en esta universidad se comunique un ethos, no solo de libertad observante (superar la falsa libertad de hacer el mal), sino de libertad expansiva (hacer rendir los propios talentos) y de liberación (la lucha personal por vencer servidumbres propias y ajenas). Tercero (en realidad, el ideal): que los cristianos seamos alter Christus (otro Cristo) para los demás. El lema plantea una universidad panamericana utópica (deslocalizada), que pretende localizarse en lugares como Mixcoac, la Bona Terra, Guadalajara..., e influir ampliamente en toda nuestra sociedad. Sin esa pretensión, no habría UP”, José Antonio Coronel Salinas, "Nuestro lema: Ubi spiritus, libertas (Educación y libertad)" (2018), 8 . 
Llano, Alejandro. "Universidad y unidad de vida según san Josemaría Escrivá”, en Documentos del Instituto de Antropología y Ética, n. ${ }^{\circ}$ 24. Pamplona: Universidad de Navarra, 2002.

Llovet Abascal, José María. “Aletheia, emet y veritas". Revista humanidades, Foro Universitas, (2013): 22-40.

Lorda, Juan Luis. La vida intelectual en la Universidad. Fundamentos, experiencias y libros. Pamplona: EUNSA, 2016.

MacIntyre, Alasdair. Dependent Rational Animals. Why Human Beings Need the Virtues. Chicago: Open Court, 1999.

Marín, Higinio. “La universidad como excepción”. En El hombre y sus alrededores.

Ediciones Cristiandad, 231 y ss, 2013.

Martínez Echevarría, Miguel Alfonso. "El modo universitario de entender la economía". Nuevas Tendencias, $\mathrm{n}^{\circ} 93$ (2014): 3-15.

Martínez Echevarría, Miguel Alfonso y Germán Scalzo. “Trabajo, don, cultura y economía: Hacia un nuevo enfoque del problema económico". Cuadernos Salmantinos de Filosofía 47, (2020): 379-399.

Mauss, Marcel. Ensayo sobre el don. Forma y función del intercambio en las sociedades arcaicas. Buenos Aires: Katz Editores, 2009.

Miguens, Fernando. Fe y cultura en la enseñanza de Juan Pablo II. Madrid: Palabra., 1994. Ocariz, Fernando. Carta pastoral. 9 de enero de 2018.

Pablo VI. Vaticano II, Constitución pastoral Gaudium et Spes. 7 de diciembre de 1965.

Pieper, Josef. Sobre los mitos platónicos. Barcelona: Herder, 1984. 
Polo, Leonardo. Quién es el hombre. Un espíritu en el tiempo. Madrid: Rialp, 1991.

Ratzinger, Joseph. Introducción al Cristianismo. Salamanca: Ediciones Sígueme, 2001.

Readings, Bill. The University in Ruins. Cambridge: Harvard University Press, 1996.

Rieff, Philip. Charisma. The Gift of Grace, and How It has Been Taken Away from Us.

New York: Pantheon Books, 2007.

Soloviov, Vladímir. La justificación del bien. Ensayo de filosofía moral. Salamanca:

Ediciones Sígueme, 2002.

Steiner, Franz. Taboo. London: Cohen and West, 1956.

Tenbruck, Friedrich. "The problem of thematic unity in the works of Max Weber".

British Journal of Sociology 31, n. ${ }^{\circ} 3$ (1980): 316-351.

Weber, Max. El político y el cientifico. Madrid: Alianza, 1967.

Weber, Max. La ética protestante y el espíritu del capitalismo.

Editado por Francisco Gil Villegas. México: Fondo de Cultura Económica, 2003. 